\title{
Analysis of electron capture at oxide traps by electric field injection
}

\author{
Olof Engstrom, ${ }^{1, a)}$ Naser Sedghi, ${ }^{2}$ Ivona Z. Mitrovic, ${ }^{2}$ and Stephen Hall ${ }^{2}$ \\ ${ }^{1}$ Department of Microtechnology and Nanoscience, Chalmers University of Technology, \\ SE-41296 Gothenburg, Sweden \\ ${ }^{2}$ Department of Electrical Engineering and Electronics, University of Liverpool, Brownlow Hill, \\ Liverpool L69 3GJ, United Kingdom
}

(Received 31 March 2013; accepted 13 May 2013; published online 30 May 2013)

Electron injection into oxide traps of metal/high- $k$ oxide/interlayer/silicon structures is investigated by modeling. We demonstrate the influence on flat-band voltage by the sharpness of the interlayer/ silicon interface and by the properties of traps in the oxide. Since charge carrier injection in this kind of structures may take place by two different processes simultaneously, excluding one or the other in the interpretation of data may lead to considerable erroneous results in extracted values of capture cross sections. (C) 2013 AIP Publishing LLC. [http://dx.doi.org/10.1063/1.4807845]

Electrical characterization of charge carrier traps in oxides of metal-oxide-silicon (MOS) structures has a long history and divides into two methodologies, which differ in the process of charge capture and are still frequently used. An early conception by Heiman and Warfield ${ }^{1}$ is based on trapping from gap states induced by the silicon crystal, ${ }^{2}$ where electrons or holes are injected into the oxide bandgap by tunneling and subsequently captured by defects. ${ }^{1,3-7}$ Another common treatment relies on Fowler-Nordheim (FN) injection or on substrate injection by hot electrons from the silicon crystal into the oxide energy bands, followed by capture into traps from this initial position. ${ }^{8-15}$ For both methods, the measured quantity is the change in flat-band voltage as a function of voltage or injected carrier concentrations. Due to the need for measuring the often fast response of this quantity, different schemes have been developed for performing the injection by gate voltage pulses on MOS capacitors and detecting the corresponding change in flat-band voltage by displacement current techniques or from the transfer characteristics of MOS transistors. ${ }^{5,7,16,17}$ Commonly, the quantities extracted from experimental data for characterizing the traps are their capture cross sections "seen" by the injected charge carriers.

Capture events occurring by these processes are not straightforwardly separated from each other. Since trapping from gap states occurs close to the semiconductor/oxide interface while trapping from the energy bands may take place across the rest or indeed across the entire insulator volume, different sections of the sample are probed in any experiment. Furthermore, separating the two processes from each other in the same sample is problematic. In spite of this interrelation, the two methods have been living side by side in the literature, seemingly without mutual contact. In the present paper, we make an analysis of the difference in measured results expected in such data, depending on whether capture occurs from induced gap states or from the energy band. We demonstrate that trapping from induced gap state injection (GI) and after injection into the oxide energy bands (BI) by FN tunneling are expected to occur simultaneously. Assumption of the predominance of a single injection process can lead to large errors in the extracted

a)olof.engstrom@chalmers.se values of capture cross sections, if not taken into account when interpreting measured data. Also, we find that the properties of interlayer/high- $k$ interfaces, present in current gate oxide technology, may have a significant influence on measured data.

Fig. 1 is a schematic depiction of the conduction bands at the interface between $n$-type silicon and a single layer oxide. A positive gate voltage is assumed to bend the silicon conduction band into accumulation such that electron injection occurs from the Fermi level, marked $E_{F}$. The dotted arrow represents the tunneling path for an electron injected from the silicon conduction band, thus, extending its state into the oxide band gap. After passing the energy level of the oxide trap, GI may result in capture within the range $x_{G I}-$ $x_{B I}$ marked in Fig. 1. Also, there exists a certain probability for BI by Fowler-Nordheim tunneling to the oxide conduction band for distances beyond $x_{B I}$, followed by electron drift by the electric field before possible capture into the traps. We will limit the present analysis to these two possibilities as reproduced in Fig. 1 and assume that all traps, positioned below the silicon Fermi-level, can capture electrons, such that $x_{G I}=0$.

In order to demonstrate the differences between trapping originating from the two injection processes, we study the

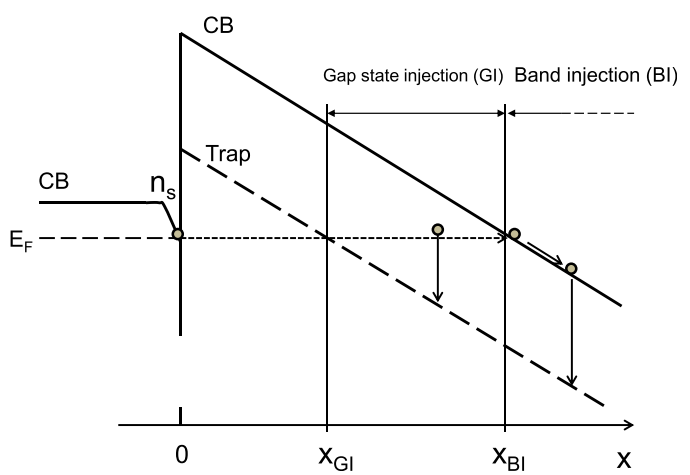

FIG. 1. Schematic illustration of the conduction band combination of a single layer oxide on silicon. Electrons are injected from the accumulated silicon interface into the oxide band gap followed by Fowler-Nordheim injection when reaching the oxide conduction band. Gap state injection occurs in the range $x_{G I}-x_{B I}$, Fowler-Nordheim injection beyond $x_{B I}$. For a trap level below the silicon Fermi level, $x_{G I}=0$. 
conditions for tunneling in two n-type silicon/ $/ \mathrm{SiO}_{2} / \mathrm{high}-k$ stacks with two different sharpness values of the $\mathrm{SiO}_{2} /$ high- $k$ interface. We take into account gradual transitions using the method published earlier in Ref. 18, where the position dependent dielectric constant, $k(x)$ was fitted to experimental literature data, as follows:

$$
k(x)=\left(k_{h}-k_{I L}\right)\left(1-\frac{1}{1+\exp \left(\frac{x-x_{F}}{x_{0}}\right)}\right)+k_{I L} .
$$

Here, $k_{h}$ and $k_{I L}$ are the dielectric constants of the high- $k$ part and $\mathrm{SiO}_{2}$, respectively. The sharpness of this interface is determined by $x_{0}$, chosen to $0.05 \mathrm{~nm}$ and $0.3 \mathrm{~nm}$ for the two cases considered below, where the former value gives rise to an "abrupt" $\mathrm{SiO}_{2} / \mathrm{HfO}_{2}$ interface, while the latter allows for a possible gradual transition between the two dielectrics. ${ }^{18}$ The interlayer thickness was taken as $x_{F}=1.5 \mathrm{~nm}$. For the total oxide thickness, $d=20 \mathrm{~nm}$ was used and the $k$-values were $k_{I L}=3.9$ and $k_{h}=25$. The band offset value between silicon and the high- $k$ oxide was set to $1.5 \mathrm{eV}$.

The result of such calculations, assuming a total voltage drop across the oxide of $V_{t}=4 \mathrm{~V}$, is shown in Fig. 2(a). Here, the conduction band edge of the oxide is shown for the two values of $x_{0}$ as a function of depth, $x$, from the silicon
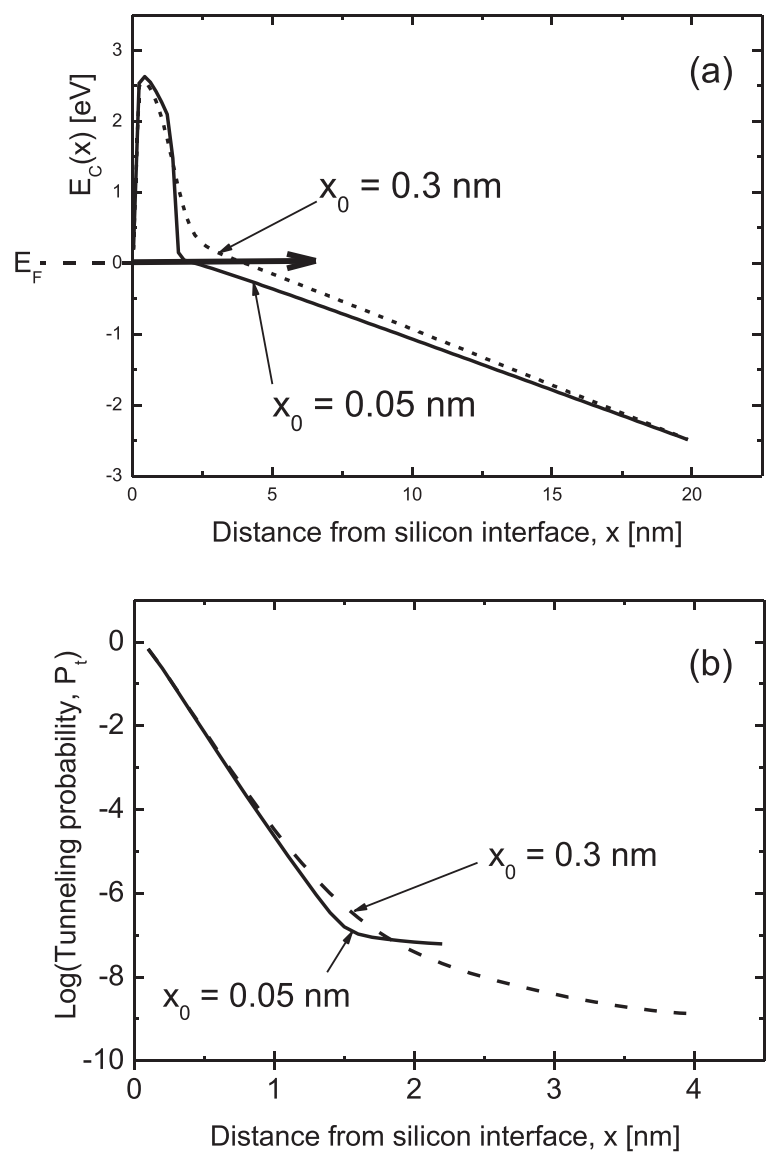

FIG. 2. (a) Oxide conduction band for a stack with $1.5 \mathrm{~nm} \mathrm{SiO}_{2}$ interlayer and an $18.5 \mathrm{~nm}$ thick high-k oxide with $k=25$, for two values of the sharpness of the interlayer/high- $k$ interface. (b) Probability $P_{t}(x)$ for injection of an electron from the silicon Fermi level position at $E_{F}$ for the two potential distributions shown in (a). Band injection occurs at the end points of the $P_{t}$ graphs, beyond which $P_{t}$ is constant. interface. Added here to the treatment in Ref. 18 is the mirror effect of an electron entering the oxide, which softens and decreases the energy barrier set up by the interlayer. Also as in Ref. 18, the variation in effective mass of the electron as a function of depth in the transition range between $\mathrm{SiO}_{2}$ and the high- $k$ oxide is used in order to calculate the tunneling probabilities, $P_{t}$, by a WKB approximation for the two $x_{0}$ values as shown in Fig. 2(b). The tunneling path from the silicon conduction band is marked by the arrow in Fig. 2(a). For $x_{0}=0.05 \mathrm{~nm}$, the interception point with the oxide conduction band, where Fowler-Nordheim injection starts, occurs close to $x=x_{F}$, while it is deeper into the high- $k$ part for the case of $x_{0}=0.3 \mathrm{~nm}$. We notice in Fig. 2(b) that reaching these points, at about $2 \mathrm{~nm}$ and $4 \mathrm{~nm}$, respectively, $P_{t}$ decreases about 7 and 9 orders of magnitude, respectively. For trap energy levels below the Fermi level of the silicon crystal, capture would occur from the induced gap state by an electron concentration determined by the injection probability, $P_{t}(x)$ as given by Fig. 2(b), whereas after crossing the conduction band edge by Fowler-Nordheim injection, the carrier concentration in the oxide conduction band is assumed constant. ${ }^{8}$

In order to demonstrate the different influences on $\Delta V_{F B}$ of the two capture processes, we choose a trap distribution of two parts, namely, one in the interlayer, close to the $\mathrm{Si} / \mathrm{SiO}_{2}$ interface and the other one deeper into the high- $k$ material as shown in Fig. 3. Comparing with Fig. 2, we notice that the distribution in the interlayer entirely gives rise to capture from induced gap states, while the deeper distribution becomes the origin of capture from the oxide conduction band. This distribution is for illustrative purposes and we do not claim it to be a particular case in a real oxide stack, although it may not be unrealistic.

The change in flat band voltage as a function of time, after switching on a voltage across the oxide depends on the injection probabilities, $P_{t}$. F

or induced gap state injection, where $P_{t}$ is a function of $x$ as shown in Fig. 2(b), the concentration, $n(x)$, of electrons, penetrating the oxide band gap from the accumulated concentration, $n_{s}$ in the silicon crystal, is $n_{s} P_{t}(x)$. From standard statistical reasoning, ${ }^{19}$ assuming that the trap energy levels are deep enough below the oxide conduction band edge for neglecting emission, we find

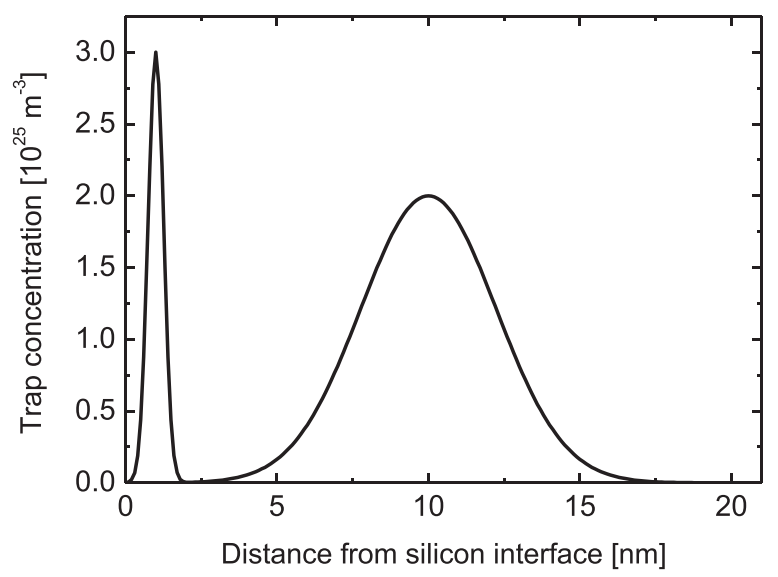

FIG. 3. Trap distribution used in the calculations. 


$$
n_{T j}(x, t)=N_{T j}(x)\left\{1-\exp \left\lfloor-n_{j}(x) v_{t h} \sigma_{j} t\right\rfloor\right\},
$$

where $j=\{G I, B I\}$ for the two injection cases, $N_{T j}$ is the concentration of traps, $v_{t h}$ is the thermal velocity of electrons in the silicon crystal and in the oxide conduction band, $\sigma_{j}$ is the capture cross section for gap state injected and band injected electrons to the traps, and $t$ is the time. The change in flat band voltage is given by

$$
\Delta V_{F B}(t)=\frac{q}{\varepsilon_{0}} \int_{0}^{x_{B I}} \frac{d-x}{k(x)} n_{T G I}(x, t) d x+\frac{q}{\varepsilon_{0}} \int_{x_{B I}}^{d} \frac{d-x}{k(x)} n_{T B I}(x, t) d x,
$$

where the first and second terms give the contribution from gap state and band state injected electrons, respectively.

Fig. 4 shows calculated results of the time evolutions, $\Delta V_{F B}(t)$, for the two cases of $x_{0}$ values with capture cross sections chosen to be $\sigma_{G I}=10^{-25} \mathrm{~m}^{2}$ and $\sigma_{B I}=10^{-21} \mathrm{~m}^{2}$ and taking $n_{s}=10^{25} \mathrm{~m}^{-3}$, which represents about $50 \%$ of the effective density of states in the silicon conduction band. We find a dissimilarity in shapes influenced by the values of $x_{0}$. A difference of two orders of magnitude in $P_{t}$ between the two cases of $x_{0}$ is observed in Fig. 2(b) at the points where
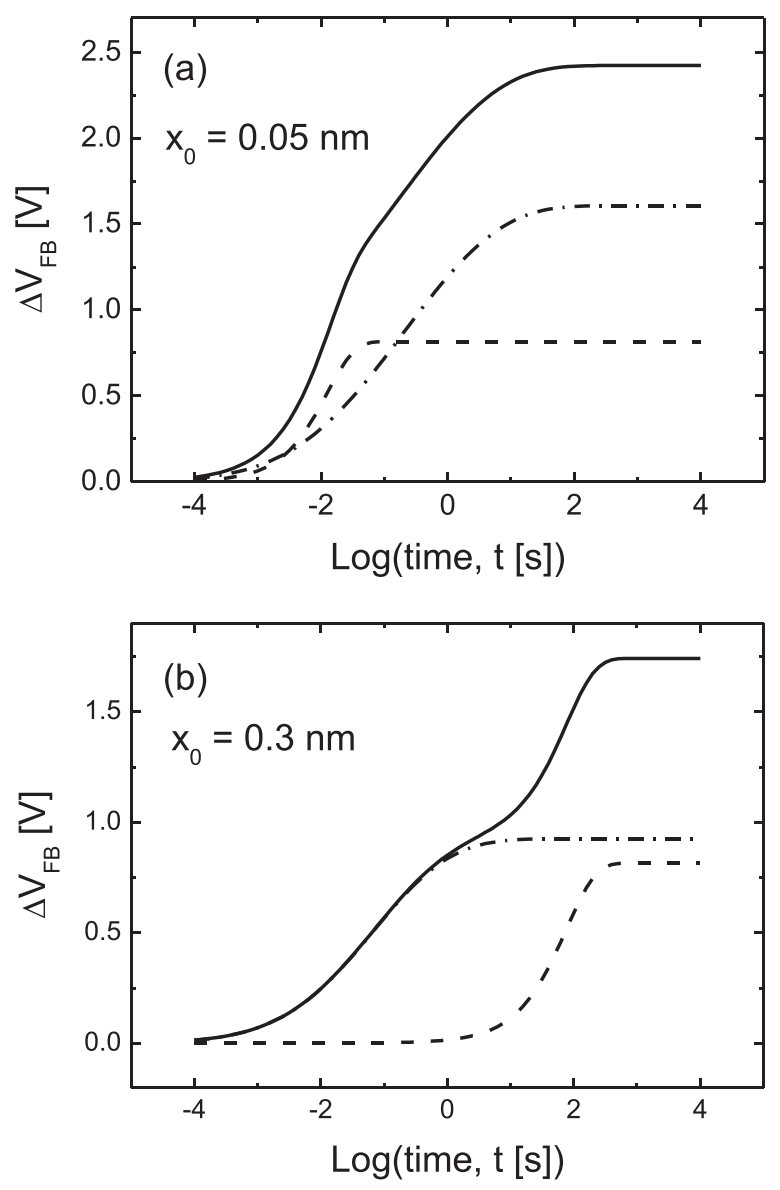

FIG. 4. Time dependence of the flat-band voltage after switching on an oxide voltage of $4 \mathrm{~V}$ across the structure in Fig. 2. Capture cross sections were set to $\sigma_{G I}=10^{-25} \mathrm{~m}^{2}$ and $\sigma_{B I}=10^{-21} \mathrm{~m}^{2}$. (a) Interlayer/high- $k$ sharpness $x_{0}=0.05 \mathrm{~nm}$. (b) Interlayer $/$ high $k$ sharpness $x_{0}=0.3 \mathrm{~nm}$. The dashed curves originate from capture into the oxide bulk and the point dashed curves from capture into the states close to the silicon interface in Fig. 3. The solid curve is the total, given by the sum of those two.
BI occurs. This gives rise to a difference of about four orders of magnitude in position on the time axis between the dashed curves, representing BI in Figs. 4(a) and 4(b) and results in two completely different characters of the total $\Delta V_{F B}(t)$ evolutions (solid curve) for the two cases of $x_{0}$. In the case of $x_{0}=0.05 \mathrm{~nm}$, the shape is dominated by GI (point dashed curve), giving a more rounded shape to the final part and a slight kink at the point where GI starts to dominate over BI. For $x_{0}=0.3 \mathrm{~nm}$, the first part of $\Delta V_{F B}(t)$ is dominated by GI while BI capture takes over at a later point of time, when capture by GI has saturated. The combination of these two effects gives rise to a characteristic kink in $\Delta V_{F B}(t)$, followed by an increased slope. Both these characteristic features have been observed in experimental literature. ${ }^{9-11,17}$ For shapes like those shown in Fig. 4(b), this has often been explained as a result of traps with two different capture cross sections in the bulk part of the oxide. Here, we notice that, even if the capture cross sections of the two trap distributions are different, their different depth positions have a crucial influence. Considering Fig. 2(b), one finds that $P_{t}$ for positions close to the silicon interface is similar for the 0.05 and $0.3 \mathrm{~nm}$ case, whereas the probability curves separate for deeper positions. This explains why the point dashed curve in Fig. 4, for the states in the interlayer, remains at about the same abscissa position while the dashed curve for the bulk states have a different position for the two cases. Moreover, one notices in Fig. 4 that the saturated values of $\Delta V_{F B}$ are larger for the case $x_{0}=0.05 \mathrm{~nm}$ than for $x_{0}=0.3 \mathrm{~nm}$. Due to the more gradual change of $k(x)$ in the latter case, the effective capacitance of this structure becomes larger, ${ }^{18}$ which gives rise to a smaller change, $\Delta V_{F B}$, for a given charge injection.

In Fig. 4(a), the saturated $\Delta V_{F B}$ value contributed by the interlayer charge is found to be about twice as large as that given by the bulk charge. Considering the large ratio in total charge of about a factor of 5 in favor of the bulk trap distribution shown in Fig. 3, the relative saturation values for their contributions to $\Delta V_{F B}$, as displayed in Fig. 4(a), may be found unexpected. This apparent oddity originates from the combination of the difference in $k$ values of the dielectrics surrounding the charge clusters and their distances, $d-x$, to the surface of the $\mathrm{HfO}_{2}$ layer, as can be understood from Eq. (3). A ratio of effective $k$-values of about 17/3.9 and the ratio of about $1 / 2$ between the positions $d-x$ of the charge centroids provide this interrelation of $\Delta V_{F B}$ values, in spite of the large difference in total charge. Similarly, when going from $x_{0}=0.05$ to $0.3 \mathrm{~nm}$, the decrease of about $30 \%$ in $\Delta V_{F B}$ contribution from the interlayer charge, seen in Fig. 4, is explained by an increased $k$ value from 3.9 to 7 at this charge position due to the gradual interface transition characterized by Eq. (1) and demonstrated by Fig. 1 in Ref. 18.

The positions along the time axis in Figs. 4(a) and 4(b) for the point dashed and the dashed curves not only depend on the injection probabilities but also the values of capture cross sections, trap positions and distributions have an influence. The value of $\sigma_{G I}=10^{-25} \mathrm{~m}^{2}$ for states in the interlayer is within the range of experimental data for interface states of $\mathrm{P}_{\mathrm{b}}$ - type $\mathrm{e}^{20}$ and states at the interlayer/ $/ \mathrm{HfO}_{2}$ interface ${ }^{21}$ and serves as a reference for the calculation. In order to obtain $\Delta V_{F B}(t)$ graphs with shapes commonly seen in experimental data, $\sigma_{B I}=10^{-21} \mathrm{~m}^{2}$ was used. This is in the lower range of 
what often is found for bulk traps. However, choosing four orders of magnitude higher value of $\sigma_{B I}$ for the case $x_{0}=0.3 \mathrm{~nm}$ in Fig. 4(b) would move the dashed curve to a position close to that of the point dashed curve, valid for GI. Hence, interpreting an experimental result as shown in Fig. 4(b) under the false assumption that the entire shape of $\Delta V_{F B}(t)$ is a result of capture by $\mathrm{BI}$ and Fowler-Nordheim tunneling would give an erroneous value of $\sigma_{B I} \approx 10^{-17} \mathrm{~m}^{2}$ instead of $10^{-21} \mathrm{~m}^{2}$ used in the calculation.

In BI experiments, the injected electron concentration $n_{B I}(x)$ in Eq. (2) is a constant, which makes it possible to determine this quantity by measuring the charge passing through the sample during a specific time interval ${ }^{8-14}$ and achieve the capture cross section for bulk traps from Eq. (3). On the other hand, for that part of $\Delta V_{F B}(t)$ data, which originates from GI, the extraction of capture cross section values requires knowledge about $P_{t}$. As realized from Fig. 4, different $\Delta V_{F B}(t)$ results are expected depending on the properties of the interlayer/high- $k$ interface. Therefore, erroneous values of capture cross sections might be extracted unless such information is known. This problem is amplified when recalling that only direct tunneling has been considered in the present description. Taking into account the alternative of trap assisted tunneling, possibly connected with the Poole-Frenkel effect for the electron supply to the oxide conduction band, will add uncertainties.

The faster approach to the saturation value for the dashed curve compared to the point dashed data in Fig. 4 is understood by the slopes of $P_{t}$ in Fig. 2(b). For the states in the interlayer, the negative $P_{t}$ slopes at GI give rise to a "travelling front" of trap filling, ${ }^{4}$ while the constant $P_{t}$ values at BI for the bulk traps brings about a more or less simultaneous filling of all traps of that ensemble. The difference in feature between the two main $\Delta V_{F B}(t)$ curves in Fig. 4 might be used as a first rough estimate to assess the relative influence between capture by GI and by BI. In most experimental situations, it seems proper to assume that one of the two injection processes seldom occurs alone.

The influence demonstrated for the change in flat-band voltage as a function of time at two quantified sharpness conditions for the interlayer/high- $k$ interface may extend beyond the limits of the two values exercised here. The values $x_{0}=0.05 \mathrm{~nm}$ and $0.3 \mathrm{~nm}$ were used in Ref. 18 and fitted to experimental data for $\mathrm{SiO}_{2} / \mathrm{HfO}_{2}$ interfaces. In practice, one would expect such properties to vary, depending on the type of high- $k$ oxide investigated and on process data. ${ }^{22}$

The aim of the present investigation is to demonstrate the relative influence of oxide interface properties, trap positions, and distributions on the extraction of capture cross sections from experimental data. Therefore, sample dependent quantities like the surface concentration, $n_{s}$, and the influence of oxide charge on electron transport after BI have been just estimated and omitted, respectively. Likewise, to predict the behavior of specific samples, a higher precision on the theoretical tunneling data than the WKB approximation used here is probably necessary.

The stability of threshold voltage as a result of gate voltage stress is an important property of MOS transistors, which makes characterization of oxide traps by electrical methods, highly desirable. For the methodology discussed in the present treatment, this requires independent information on specific trap properties: energy positions, atomic relaxation, ${ }^{23,24}$ concentration profiles, and oxide interface properties. The influence on flat-band voltage shifts by such materials properties must be known before more precise conclusions on the properties of traps capturing charge in MOS structures can be extracted from experimental data based on the time dependence of flat-band voltage.

${ }^{1}$ F. P. Heiman and G. Warfield, IEEE Trans. Electron Devices 12, 167 (1965).

${ }^{2}$ W. Mönch, J. Appl. Phys. 109, 113724 (2011).

${ }^{3}$ I. Lundström, S. Christensson, and C. Svensson, Phys. Status Solidi A 1, 395 (1970).

${ }^{4}$ D. J. Breed, Solid-State Electron. 17, 1229 (1974).

${ }^{5}$ C. Leroux, J. Mitard, G. Ghibaudo, X. Garros, G. Reimbold, B. Guillamot, and F. Martin, Tech. Dig. - Int. Electron Devices Meet. 2004, 737.

${ }^{6}$ S. Hall and O. Buiu, IEEE Trans. Electron Devices 54, 272 (2007).

${ }^{7}$ M. Cho, R. Degrave, P. Roussel, B. Govoreanu, B. Kaczer, M. B. Zahid, E. Simoen, A. Arreghini, M. Jurczak, J. Van Houdt, and G. Groseneken, Solid-State Electron. 54, 1384 (2010).

${ }^{8}$ T. H. Ning, J. Appl. Phys. 47, 3203 (1976).

${ }^{9}$ J. F. Zhang, S. Taylor, and W. Eccleston, J. Appl. Phys. 71, 725 (1992).

${ }^{10}$ S. Zafar, A. Callegeri, E. Gusev, and M. V. Fischetti, J. Appl. Phys. 93, 9298 (2003)

${ }^{11}$ C. Z. Zhao, J. F. Zhang, M. B. Zahid, B. Govoreanu, G. Groeseneken, and S. De Gent, J. Appl. Phys. 100, 093716 (2006).

${ }^{12}$ M. H. Chang, J. F. Zhang, and W. D. Zhang, IEEE Trans. Electron Devices 53, 1347 (2006).

${ }^{13}$ M. Hara, Y. Okamoto, and H. Ohnuma, Jpn. J. Appl. Phys., Part 1 9, 1103 (1970).

${ }^{14}$ J. F. Verwey, J. Appl. Phys. 44, 2681 (1973).

${ }^{15}$ T. H. Ning and H. N. Yu, J. Appl. Phys. 45, 5373 (1974).

${ }^{16}$ R. Rao, R. Simoncini, H. D. B. Gottlob, M. Schmidt, and F. Irrera. J. Vac. Sci. Technol. B 29, 01A902-1 (2011).

${ }^{17}$ N. Sedghi, I. Z. Mitrovic, S. Hall, J. M. J. Lopes, and J. Schubert, J. Vac. Sci. Technol. B 29, 01AB03-1 (2011).

${ }^{18}$ O. Engström, I. Z. Mitrovic, and S. Hall, Solid-State Electron. 75, 63 (2012).

${ }^{19}$ C. T. Sah, L. Forbes, I. Rosier, and A. F. Tasch, Jr., Solid-State Electron. 13, 759 (1970).

${ }^{20}$ J. Piscator, B. Raeissi, and O. Engstrom, J. Appl. Phys. 106, 054510 (2009).

${ }^{21}$ B. Raeissi, J. Piscator, Y. Y. Chen, and O. Engstrom, J. Electrochem. Soc. 158, G63 (2011).

${ }^{22}$ I. Z. Mitrovic, S. Hall, N. Sedghi, G. Simutis, V. R. Dhanak, P. Bailey, T. C. Q. Noakes, I. Alexandrou, O. Engstrom, J. M. J. Lopes, and J. Schubert, J. Appl. Phys. 112, 044102 (2012).

${ }^{23}$ K. Xiong, J. Robertson, M. C. Gibson, and S. J. Clark, Appl. Phys. Lett. 87, 183505 (2005).

${ }^{24}$ J. L. Gavartin, D. Ramo Munos, A. L. Schluger, G. Bersuker, and B. H. Lee, Appl. Phys. Lett. 89, 082908 (2006). 